\title{
Prodigiosin biosynthesis gene cluster in the roseophilin producer Streptomyces griseoviridis
}

\author{
Takashi Kawasaki, Fumi Sakurai, Shun-ya Nagatsuka and Yoichi Hayakawa
}

\begin{abstract}
Streptomyces griseoviridis $2464-\mathrm{S} 5$ produces prodigiosin R1, a tripyrrole antibiotic, and roseophilin, a structurally related compound containing two pyrrole and one furan rings. A gene cluster for the biosynthesis of a prodigiosin was identified in $S$. griseoviridis. The cluster consisted of 24 open reading frames, including 21 genes ( $r p h D-r p h Z)$ homologous to prodigiosin biosynthesis genes in the red cluster in Streptomyces coelicolor A3(2). The expression of $r p h N$ in S. coelicolor lacking redN restored the production of prodigiosin.
\end{abstract}

The Journal of Antibiotics (2009) 62, 271-276; doi:10.1038/ja.2009.27; published online 27 March 2009

Keywords: biosynthesis gene; prodigiosin; roseophilin; Streptomyces griseoviridis

\section{INTRODUCTION}

Several tripyrrole antibiotics are known to belong to the prodigiosin family. ${ }^{1-4}$ S. griseoviridis $2464-\mathrm{S} 5$ produces prodigiosin $\mathrm{R} 1^{5}$ and roseophilin, ${ }^{6}$ a structurally related compound containing two pyrrole and one furan rings, as shown in Figure 1. Roseophilin is considered to be biosynthesized partially with the same pathway as prodigiosin R1. Prodigiosin-family biosynthesis genes have been reported to exist in the pig gene clusters in Serratia marcescens ATCC 274 and Serratia sp. ATCC 39006 (which make prodigiosin itself), ${ }^{7}$ in the red gene cluster in S. coelicolor A3(2) (which makes undecylprodiginine) ${ }^{8}$ and in the hap gene cluster in Hahella chejuensis KCTC 2396 (which makes prodigiosin itself). ${ }^{9}$ PCR amplification of $S$. griseoviridis genomic DNA has identified genes homologous to redH, redM and redW, involved in the biosynthesis of undecylprodiginine in S. coelicolor (Figure 2). ${ }^{5}$ We report herein the cloning and characterization of the $r p h$ gene cluster for prodigiosin biosynthesis from S. griseoviridis 2464-S5.

\section{RESULTS}

Amplification of prodigiosin biosynthesis genes from S. griseoviridis

In the biosynthesis of prodigiosin, the redN/pigH gene product is involved in the formation of the central pyrrole moiety (Figure 2). As roseophilin contains a furan ring in place of the central pyrrole ring in prodigiosins, redN/pigH might be characteristic of the prodigiosin biosynthesis. A pair of primers was designed from the conserved amino acid (aa) sequences of redN/pigH in the three prodigiosin producers, S. coelicolor A3(2), Serratia marcescens ATCC 274 and Serratia sp. ATCC 39006. ${ }^{7,8}$ PCR amplification of $S$. griseoviridis genomic DNA gave a redN/pigH-like gene fragment. Nucleotide sequencing and homology search showed that the fragment shared amino acid identity of $84 \%$ with RedN.
The prodigiosin biosynthesis genes, redM and redW, encode L-prolyl-AMP ligase and L-prolyl-PCP dehydrogenase, respectively (Figure 2). These two enzymes have been reported to be commonly involved in the biosynthesis of pyrrole-containing antibiotics. ${ }^{10}$ PCR was carried out using several primers designed from the conserved amino acid sequences of L-prolyl-AMP ligase and L-prolyl-PCP dehydrogenase genes in the coumermycin $A_{1}$ producer, Streptomyces rishiriensis DSM 40489 (proB and proA), ${ }^{11}$ in the pyrrolomycin producers, Streptomyces vitaminophilum ATCC 31673 (pyr8 and pyr7) and Streptomyces sp. Strain UC 11065 (dox8 and dox7) ${ }^{12}$ and in the prodigiosin producer, S. coelicolor A3(2) (redM and red W). DNA sequencing of the PCR products identified single genes encoding L-prolyl-AMP ligase and L-prolyl-PCP dehydrogenase in S. griseoviridis.

Southern hybridization of genomic DNA with prodigiosin biosynthesis genes

Southern blot analysis was carried out on S. griseoviridis genomic DNA to identify redH-, redM-, redN- and redW-like genes. Digested genomic DNA with BamHI in agarose gel was hybridized with the PCR products as probes. Each single band was detected by Southern hybridization as shown in Figure 3, suggesting that the strain contained a single set of genes for prodigiosin/roseophilin biosynthesis.

Cloning of a prodigiosin biosynthesis gene cluster from S. griseoviridis

A cosmid clone was selected by colony hybridization and Southern blot analysis from a cosmid library using the redW-like gene fragment as a probe. Nucleotide sequencing identified genes homologous to redY, redX and redD, as well as redW, in a SacI 6.0-kbp fragment of the cosmid (Figure 4). Further Southern hybridization was carried out on 
<smiles>CCCCCC1=C/C(=C/c2[nH]c(-c3ccc[nH]3)cc2OC)N=C1C</smiles>

Prodigiosin

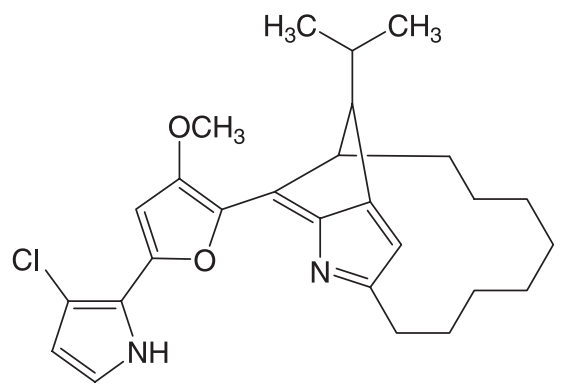

Roseophilin<smiles>CCCCCCCCCCCC1=NC(=Cc2[nH]c(-c3ccc[nH]3)cc2OC)C=C1</smiles>

Undecylprodiginine<smiles>CCC(C=C1C2=N/C(=C\c3[nH]c(-c4ccc[nH]4)cc3OC)C1CCCCCCCC2)CC(C)C</smiles>

Prodigiosin R1<smiles>CCCCC1CCCCCCC2=NC(=Cc3[nH]c(-c4ccc[nH]4)cc3OC)C1=C2</smiles>

Butyl-meta-cycloheptylprodiginine

Figure 1 Structures of roseophilin and prodigiosins.

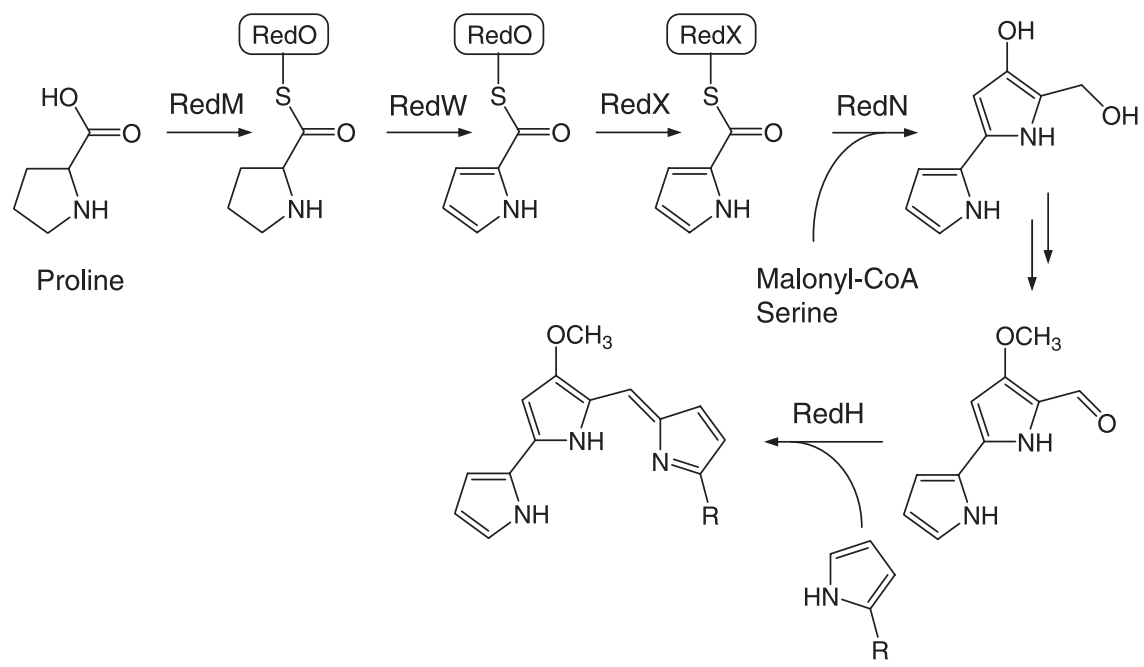

Figure 2 Proposed pathway of prodigiosin biosynthesis.

various cosmid fragments using the redH-, redM- and redN-like gene fragments as probes. Selected cosmid fragments were sequenced to identify a gene cluster containing 24 open reading frames (ORFs) as shown in Figure 4. Homology search showed that 21 of the 24 ORFs were homologous to the red genes involved in the biosynthesis of undecylprodiginine and butyl-meta-cycloheptylprodiginine in 


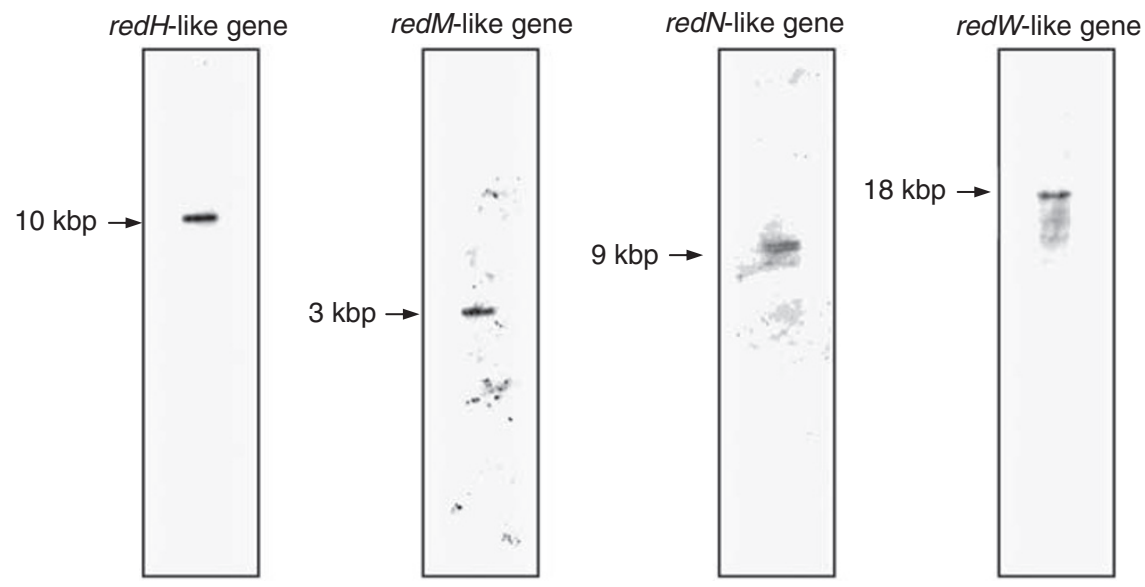

Figure 3 Southern blot analysis of S. griseoviridis genomic DNA.

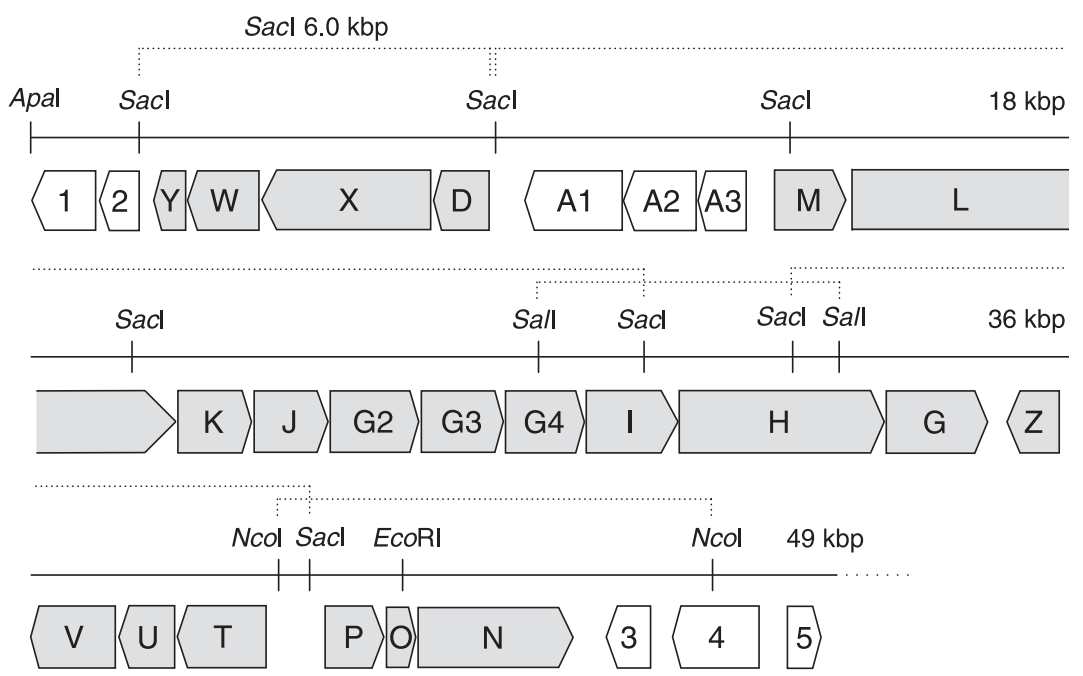

Figure 4 Prodigiosin biosynthesis gene cluster ( $r$ hh cluster) from S. griseoviridis.

S. coelicolor $\mathrm{A} 3(2),{ }^{8}$ and the corresponding genes in S. griseoviridis were designated as $r p h D-r p h Z$ (Table 1). In the cluster, sequence homology of rphA1 and rphA2 to ABC-transporter genes assigned them to self-resistance genes. A regulatory gene, $r p h A 3$, was also identified from sequence homology to a transcriptional regulator gene in Mycobacterium ulcerans (Table 1).

\section{Expression of $r p h N$ in $S$. coelicolor lacking redN}

To establish the function of the $r p h$ genes, we disrupted the red $N$ gene in $S$. coelicolor A3(2). The disruption plasmid, pRedN-DIS, was constructed (Figure 5) and introduced into S. coelicolor. Three colorless colonies were selected on R2YE plates and the redN gene disruption was confirmed by PCR analysis using their genomic DNAs as templates.

The $r p h N$ expression vector, pWHM-rphN, was constructed and introduced into $S$. coelicolor lacking redN (S. coelicolor $\Delta$ redN). S. coelicolor $\Delta$ redN, harboring pWHM-rphN or an empty vector (pWHM860), and the wild-type strain were cultivated and the mycelial extracts were analyzed by HPLC. The redN disruptant expressing $r p h N$ produced undecylprodiginine, a major prodigiosin from the wild type (Figure 6), thereby showing that $r p h N$ can act as a prodigiosin biosynthesis gene in $S$. coelicolor.

\section{DISCUSSION}

The $r p h$ gene cluster consisted of 21 genes homologous to prodigiosin biosynthesis genes in $S$. coelicolor, two self-resistance genes and a regulatory gene. The putative functions and locations of orf 1 (DNA helicase) and orf3 (RNA methyltransferase) suggested that orf1, orf2, orf 3 , orf4 and orf5 are excluded from the biosynthesis gene cluster (Table 1 and Figure 4). The rph cluster did not contain genes homologous to redC, redE, redF, redQ, redR or redS in S. coelicolor (Figure 7). Among them, redQ and redR have been reported to be involved in the biosynthesis of an alkyl side chain and not to be essential for the biosynthesis of undecylprodiginine. ${ }^{13}$ Although the redF-homologous gene, pigN, was involved in $\mathrm{O}$-methylation, two pigN mutants reduced, but retained, prodigiosin productivity. ${ }^{14}$ No role has been assigned to redS in the prodigiosin pathway and only the 146 aa $N$-terminal region of PigB (671 aa) shows sequence similarity with RedS. ${ }^{7}$ In Serratia sp., PigB catalyzes the formation of 2-methyl-3- $n$-amylpyrrole (MAP), which is replaced by 2-undecylpyrrole in S. coelicolor. ${ }^{14}$ No role has been assigned to redC or to redE, and no homologous genes have been found in the pig cluster in Serratia sp. ${ }^{7}$ These findings suggest that these genes are nonessential for the biosynthesis of prodigiosins, and the $r p h$ gene cluster identified here contains all the genes for prodigiosin biosynthesis in 
Table 1 Deduced functions of gene products in the $r p h$ cluster

\begin{tabular}{|c|c|c|}
\hline $\begin{array}{l}\text { Gene } \\
\text { products }\end{array}$ & Homologous protein & $\begin{array}{c}\text { Identity } \\
(\%)\end{array}$ \\
\hline Orf1 & ATP-dependent DNA helicase of Streptomyces avermitilis & 77 \\
\hline Orf2 & Uncharacterized protein of $S$. avermitilis & 47 \\
\hline RphY & Hypothetical protein (RedY) & 55 \\
\hline RphW & L-Prolyl-PCP dehydrogenase (RedW) & 69 \\
\hline RphX & $\beta$-Ketomyristoyl-ACP synthase (RedX) & 47 \\
\hline RphD & Transcriptional regulator (RedD) & 61 \\
\hline RphA1 & ABC-transporter of Mycobacterium ulcerans & 46 \\
\hline RphA2 & ABC-transporter of $M$. ulcerans & 57 \\
\hline RphA3 & Transcriptional regulator of $M$. ulcerans & 40 \\
\hline RphM & L-Prolyl-AMP ligase (RedM) & 54 \\
\hline RphL & Polyketide synthase (RedL) & 54 \\
\hline RphK & Oxidoreductase (RedK) & 68 \\
\hline RphJ & Thioesterase (RedJ) & 58 \\
\hline RphG2 & Oxygenase (RedG) & 51 \\
\hline RphG3 & Oxygenase (RedG) & 39 \\
\hline RphG4 & Oxygenase (RedG) & 44 \\
\hline Rphl & O-methyltransferase (RedI) & 59 \\
\hline $\mathrm{RphH}$ & Pyrrole-condensing enzyme (RedH) & 59 \\
\hline RphG & Oxygenase (RedG) & 57 \\
\hline RphZ & Response regulator (RedZ) & 50 \\
\hline RphV & Dehydrogenase (RedV) & 42 \\
\hline RphU & Phosphopantetheinyl transferase (RedU) & 46 \\
\hline RphT & Hypothetical protein (RedT) & 63 \\
\hline RphP & 3-Oxoacyl-ACP synthase (RedP) & 58 \\
\hline RphO & Peptidyl carrier protein (RedO) & 75 \\
\hline RphN & 4-Hydroxy-2,2'-bipyrrole-4-methanol synthase (RedN) & 78 \\
\hline Orf3 & RNA methyltransferase of Brucella abortus & 31 \\
\hline Orf4 & Uncharacterized protein of Streptomyces coelicolor & 54 \\
\hline Orf5 & Integral membrane protein of $S$. coelicolor & 63 \\
\hline
\end{tabular}

S. griseoviridis. Moreover, primers designed from redS/PigB and redF/ $p i g N$ did not amplify $S$. griseoviridis genomic DNA (data not shown).

The $r p h$ cluster included four putative oxygenase genes $(r p h G$, rphG2, rphG3 and rphG4) homologous to redG, which was assumed to catalyze the cyclization of an alkyl side chain for the biosynthesis of butyl-meta-cycloheptylprodiginine. ${ }^{8}$ The same function is required by the high-sequence similarity of $r p h G$ and red $G$ and their locations (Table 1 and Figure 7). The difference in the number and position of carbon-carbon bridges between prodigiosin R1 and roseophilin might be explained by the presence of multiple oxygenase genes.

The rph cluster did not contain biosynthesis genes characteristic of roseophilin. Such genes are involved in the furan ring formation and chlorination. The PCR and Southern hybridization analyses, however, detected single genes homologous to redH, redM and redW, which are considered to be common to both prodigiosin and roseophilin biosynthesis. These results suggested the existence of another gene cluster to complement the biosynthesis of roseophilin. Search for the second gene cluster for the roseophilin biosynthesis is in progress.

\section{METHODS}

Bacterial strains, plasmids and culture conditions

S. griseoviridis 2464-S5 and S. coelicolor A3(2) were used as sources of total DNA for the cloning experiment. Media and growth conditions for S. griseoviridis were described earlier. ${ }^{6}$ Prodigiosin production by $S$. coelicolor was assessed on R2YE plates. ${ }^{15}$ Yeast extract malt extract (YEME) liquid culture was used for genomic DNA isolation and for making protoplasts from S. coelicolor. ${ }^{15}$ Escherichia coli XL1-blue MRF' and the plasmids, pGEM-5Z,

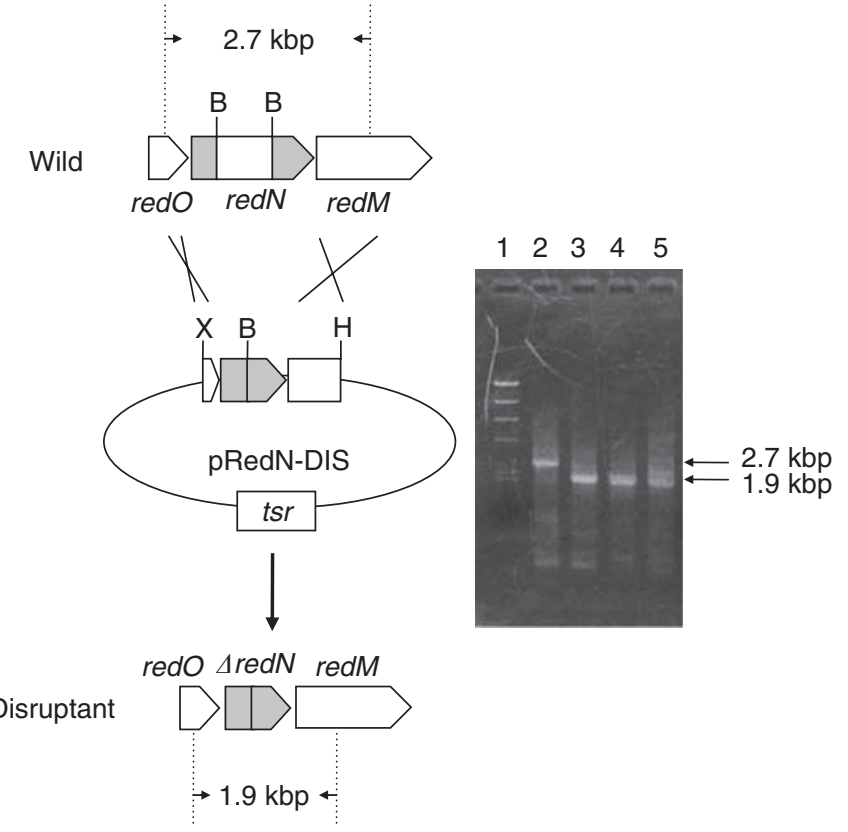

Figure 5 Disruption of the redN gene in $S$. coelicolor. Molecular marker (lane 1), PCR product from the wild-type strain (lane 2) and PCR products from the redN disruptants (lane 3-5). Amplified fragments are shown schematically. Arrows indicate primers used for PCR analysis. B: Bg/ll site. $\mathrm{X}$ : Xbal site. $\mathrm{H}$ : Hindll site.

Wild type

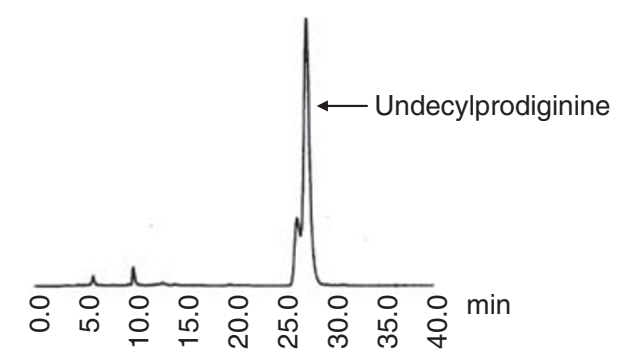

$\Delta$ redN+pWHM860
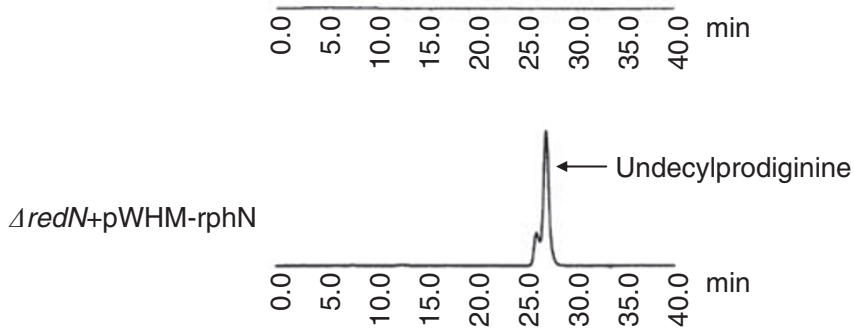

Figure $6 \mathrm{HPLC}$ analysis of prodigiosins produced by the redN disruptant expressing $r p h N$.

pGEM-7Z, pGEM-11Z, pUC118 and pUC119 were used for sequencing analyses.

DNA isolation and manipulation

All restriction enzymes, T4 DNA ligase and calf intestinal alkaline phosphatase were obtained from Nippon Gene (Toyama, Japan). Transformation of E. coli 
with plasmid DNA by electroporation was carried out under standard conditions using a Gene Pulser II electroporation system (Bio-Rad, Hercules, CA, USA). Other general procedures were carried out as described by Sambrook et al. ${ }^{16}$

\section{Amplification and cloning of prodigiosin biosynthesis genes}

A redN-like gene fragment was amplified using $S$. griseoviridis genomic DNA and a pair of primers (5'-GAYGGSGTSTTYWSSATGCAYGG- $3^{\prime}$ and $5^{\prime}$-SGGRWASACSACSGTYTGRCA- ${ }^{\prime}$ ). The reaction mixture consisted of $1 \times$ GC buffer I (TaKaRa, Kyoto, Japan), 0.4 mm dNTP (deoxyribonucleotide triphosphate) mixture, $2 \mu \mathrm{M}$ of each primer, $0.5 \mathrm{ng}^{-1} \mathrm{l}^{-1}$ genomic DNA and

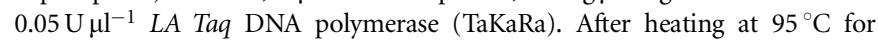
$4 \mathrm{~min}, \mathrm{PCR}$ was carried out for $30 \mathrm{cycles}\left(95^{\circ} \mathrm{C} 1 \mathrm{~min}, 50^{\circ} \mathrm{C} 30 \mathrm{~s}, 72^{\circ} \mathrm{C} 45 \mathrm{~s}\right)$. After purification with QIAquick Gel Extraction Kit (Qiagen, Hilden, Germany), the PCR products were linked with pGEM-T Easy Vector (Promega, Madison, WI, USA) using a T4 DNA ligase. E. coli was transformed with the plasmid DNA and selected clones were used for sequencing analysis.

Three sets of primers (N1: $5^{\prime}$-CTSTACACSTCSGGSWSSACSGG- ${ }^{\prime}$; C1: 5'-GTGCASACGTTSGTCTCSGTSGG-3', C2: 5' ${ }^{\prime}$-AGSRCSGASGGSACSGARTA CCA-3', C3: 5'-AGSRCGCTSGGSACSGARTACCA-3') were used to amplify L-prolyl-AMP ligase gene fragments. Various pairs of primers (N1: $5^{\prime}$-TGG GAGMGSRCSTGCCT-3', N2: 5'-CAGGCSRTSTCSCACMGSMT-3', N3: 5'-CA GGCSRTSAGCCACMGSMT-3'; C1: 5'-ATSTCSSWSGTSCCSGAGAADAT-3', C2: 5'-ATSTCSSWSGTSCCGCTGAADAT-3', C3: 5'-ATSTCSKTSGTSCCSG AGAADAT-3', C4: 5'-ATSTCSKTSGTSCCGCTGAADAT-3') were used for the amplification of L-prolyl-PCP dehydrogenase gene fragments. PCR and cloning were carried out as described above.

\section{Southern blot analysis of genomic DNA}

Gene fragments homologous to redH, redM and redW have been amplified earlier by PCR from the roseophilin producer. ${ }^{5}$ DNA fragments used as probes were labeled using the AlkPhos Direct labeling kit (GE Healthcare, Piscataway, NJ, USA). S. griseoviridis genomic DNA was digested with Bam HI and run on a $0.7 \%$ agarose gel. The DNA was transferred to nylon membranes and fixed. After the membranes were incubated with probes at $65^{\circ} \mathrm{C}$ for $16 \mathrm{~h}$, they were washed and then developed using the ECF detection kit (GE Healthcare). Images were obtained with an FLA2000 image reader (Fujifilm, Tokyo, Japan).

\section{Cloning of a prodigiosin biosynthesis gene cluster}

A cosmid library was constructed from the roseophilin producer. Genomic DNA of S. griseoviridis 2464-S5 was partially digested with Sau3AI and ligated to the pWE15 cosmid vector (Stratagene, La Jolla, CA, USA). The ligated DNAs were packaged using Gigapack III XL Packaging Extract (Stratagene) and used to infect E. coli XL1-blue MRF. E. coli colonies obtained by transfection with the cosmids were then screened for the presence of a redW-like gene by colony hybridization, using the PCR product labeled with the AlkPhos Direct labeling kit (GE Healthcare) as a probe. Selected positive clones were subsequently analyzed by Southern hybridization with the same probe, and cosmid 31 was unambiguously found to contain the redW-like sequence. A SacI 6.0-kbp fragment of the cosmid was sequenced to identify genes homologous to redD, redX, redW and redY. To isolate the biosynthesis gene cluster, cosmids were selected using redM-, redH- and redN-like genes as probes, and DNA sequencing was carried out using a SacI 21.0-kbp fragment of cosmid16, a SalI 5.0-kbp fragment of cosmid4, a SacI 8.0-kbp fragment of cosmid10 and a NcoI 7.0-kbp fragment of cosmid11 (Figure 4).

\section{Gene disruption in S. coelicolor}

The disruption plasmid, pWHM3-DIS, was based on pWHM3, an E. coli/ Streptomyces shuttle vector $(7.2 \mathrm{kbp})$ containing a thiostrepton-resistant gene..$^{15}$ pWHM3 was digested with $B c l$ I and a 1.4-kbp fragment containing a part of the replication region in Streptomyces was removed. The remaining two BclI fragments ( $4.7 \mathrm{kbp}$ and $1.1 \mathrm{kbp}$ ) were ligated to construct pWHM3-DIS.

The $\operatorname{red} N$ gene was disrupted by a double crossover event (Figure 5). A $2.7 \mathrm{kbp} \mathrm{XbaI/HindIII} \mathrm{fragment} \mathrm{of} \mathrm{redN} \mathrm{contained} \mathrm{both} \mathrm{the} \mathrm{upstream} \mathrm{and}$ downstream regions. The redN gene fragment was amplified by PCR with S. coelicolor genomic DNA and one set of primers with an additional restriction site (5'-TGCTCTAGATTCGACCTCGTCCACTACCTGCAG-3' and 5'-ACC AAGCTTGTCGCCGGGGGTGAGGGAGGGCAG- $3^{\prime}$ ). The PCR product and $\overline{X b a I / H i n} d I I I-$ digested pGEM-11Z were ligated and introduced into E. coli. A plasmid carrying the appropriate DNA fragment was selected. After sequence confirmation, the plasmid was digested with BglII. A $0.7 \mathrm{kbp}$ BglII DNA fragment in the redN gene was removed and the remaining 5.0-kbp fragment containing pGEM-11Z was self-ligated. A $2.0 \mathrm{kbp} \mathrm{XbaI/HindIII} \mathrm{fragment}$ obtained from the plasmid was subcloned into the same site of pWHM3DIS to give pRedN-DIS.

The disruption plasmid, pRedN-DIS, was introduced into S. coelicolor, and thiostrepton-resistant colonies were selected. After the selected colonies were cultivated for 3 days, an aliquot of the culture was plated on ATCC medium \#5 plates to obtain spores. Diluted spores were plated on ATCC medium \#5 plates and thiostrepton-sensitive colonies were selected. Genomic DNA was prepared from the transformant and subjected to PCR analysis with the same primers that were used to amplify a $2.7 \mathrm{kbp}$ DNA fragment of the redN gene.

\section{Expression of $r p h N$ in the redN disruptant}

To express the $r p h N$ gene in the redN disruptant, S. coelicolor $\Delta r e d N$, the $r p h N$ carrying plasmid, pWHM-rphN, was constructed. To obtain the entire $r p h N$ gene, PCR amplification was carried out under a standard condition with KOD-DNA polymerase (Toyobo, Osaka, Japan) using a pair of primers with an additional restriction site (5'-TGCTCTAGAATGACCCACATCATGACCGACCGT- $3^{\prime}$ and $5^{\prime}$-ACCAAGCTTTCAGGCCGGATCGAGGGGGACGCC-3'). After sequence confirmation, the XbaI/HindIII DNA fragment was inserted into the same sites of pWHM860 ${ }^{17}$ to give pWHM-rphN, in which the $r p h N$ gene was expressed under the control of the ermE $E^{\star}$ promoter.

pWHM-rphN was introduced into S. coelicolor $\Delta r e d N$, and the transformants were cultivated at $27^{\circ} \mathrm{C}$ for 6 days on a rotary shaker in $500-\mathrm{ml}$ Erlenmeyer flasks containing $100 \mathrm{ml}$ of production medium. The medium

\section{S. coelicolor A3(2)}

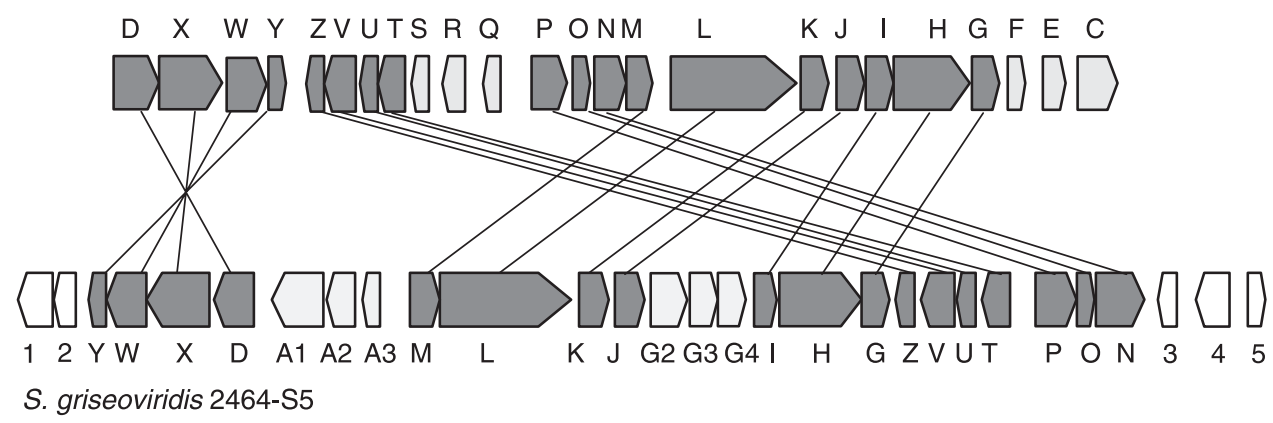

Figure 7 Comparison of the prodigiosin biosynthesis gene clusters between S. coelicolor (red) and S. griseoviridis (rph). 
contained sucrose $103 \mathrm{~g}$, glucose $10 \mathrm{~g}$, yeast extract (Difco, Detroit, MI, USA) $5 \mathrm{~g}$, Casamino acids (Difco) $0.1 \mathrm{~g}$, sodium hydrogen L-glutamate monohydrate $3 \mathrm{~g}, \mathrm{~K}_{2} \mathrm{SO}_{4} 0.25 \mathrm{~g}, \mathrm{MgCl}_{2} \cdot 6 \mathrm{H}_{2} \mathrm{O} 10.12 \mathrm{~g}, \mathrm{NaNO}_{3} 0.06 \mathrm{~g}$, TES ( $N$-tris(hydroxymethyl)methyl-2-aminoethanesulfonic acid) buffer $5.73 \mathrm{~g}, 0.5 \% \mathrm{KH}_{2} \mathrm{PO}_{4}$ $10 \mathrm{ml}, 5 \mathrm{M} \mathrm{CaCl} 24 \mathrm{ml}, 1 \mathrm{M} \mathrm{NaOH} 7 \mathrm{ml}$ and a trace element solution of $2 \mathrm{ml}$ in $1000 \mathrm{ml}$ distilled water $(\mathrm{pH} \mathrm{7.2)}$. The trace element solution consisted of $\mathrm{ZnCl}_{2} 0.004 \%, \mathrm{FeCl}_{2} \cdot 6 \mathrm{H}_{2} \mathrm{O} 0.02 \%, \mathrm{CuCl}_{2} \cdot 2 \mathrm{H}_{2} \mathrm{O} 0.001 \%, \mathrm{MnCl}_{2} .4 \mathrm{H}_{2} \mathrm{O} 0.001 \%$, $\mathrm{Na}_{2} \mathrm{~B}_{4} \mathrm{O}_{7} \cdot 10 \mathrm{H}_{2} \mathrm{O} 0.001 \%$ and $\left(\mathrm{NH}_{4}\right)_{6} \mathrm{Mo}_{7} \mathrm{O}_{24} \cdot 4 \mathrm{H}_{2} \mathrm{O} 0.001 \%$. The fermentation broth $(100 \mathrm{ml})$ was centrifuged and mycelium was extracted with acetone. After evaporation, an aqueous concentrate was extracted with ethyl acetate. The extract was dissolved in methanol and the productivity of prodigiosins was examined by reversed-phase HPLC (Senshu Pak PEGASIL ODS 4.6 i.d. $\times 250 \mathrm{~mm}$, Senshu Scientific, Tokyo, Japan) with methanol-acetonitrile-trifluoroacetic acid-water (80:3:0.15:17) at a flow rate of $1.0 \mathrm{ml} \mathrm{min}$. The corresponding peak was detected at $530 \mathrm{~nm}$.

\section{Nucleotide sequence and accession number}

DNA sequencing was carried out with an automatic DNA sequencer (3100 Genetic Analyzer, Applied Biosystems, Foster City, CA, USA). Nucleotide sequences reported here have been deposited in the GenBank, DDBJ and EMBL databases under accession number AB469822 ( $r p h$ gene cluster).

\section{ACKNOWLEDGEMENTS}

We thank Dr T Dairi of the Toyama Prefectural University for the generous gift of plasmids, E. coli strains and S. coelicolor A3(2). This work was supported in part by a Grant-in-Aid for Scientific Research, The Ministry of Education, Science, Sports and Culture, Japan.

1 Rapoport, H. \& Holden, K. G. The synthesis of prodigiosin. J. Am. Chem. Soc. 84, 635-642 (1962).

2 Gerber, N. N. \& Stahly, P. D. Prodiginine (prodigiosin-like) pigments from Streptoverticillium rubrireticuli, an organism that causes pink staining of polyvinyl chloride. Appl. Microbiol. 30, 807-810 (1975).
3 Tsao, S-W., Rudd, B. A. M., He, X-G., Chang, C-J. \& Floss, H. G. Identification of a red pigment from Streptomyces coelicolor $\mathrm{A} 3(2)$ as a mixture of prodigiosin derivatives. J. Antibiot. 38, 128-131 (1985).

4 Song, J-M. et al. Purification and characterization of prodigiosin produced by integrated bioreactor from Serratia sp. KH-95. J. Biotechnol. Bioeng. 101, 157-161 (2006).

5 Kawasaki, T., Sakurai, F. \& Hayakawa, Y. A prodigiosin from the roseophilin producer Streptomyces griseoviridis. J. Nat. Prod. 71, 1265-1267 (2008).

6 Hayakawa, Y., Kawakami, K., Seto, H. \& Furihata, K. Structure of a new antibiotic, roseophilin. Tetrahedron Lett. 33, 2701-2704 (1992).

7 Harris, A. K. P. et al. The Serratia gene cluster encoding biosynthesis of red antibiotic, prodigiosin, shows species- and strain-dependent genome context variation. Microbiology 150, 3547-3560 (2004).

8 Cerdeno, A. M., Bibb, M. J. \& Challis, G. L. Analysis of the prodiginine biosynthesis gene cluster of Streptomyces coelicolor A3(2): new mechanisms for chain initiation and termination in modular multienzymes. Chem. Biol. 8, 817-829 (2001).

$9 \mathrm{Kim}$, D. et al. Analysis of a prodigiosin biosynthetic gene cluster from the marine bacterium Hahella chejuensis KCTC 2396. J. Microbiol. Biotechnol. 16, 1912-1918 (2006).

10 Thomas, M. G., Burkart, M. D. \& Walsh, C. T. Conversion of L-proline to pyrrolyl-2carboxyl-S-PCP during undecylprodigiosin and pyoluteorin biosynthesis. Chem. Biol. 9, 171-184 (2002).

11 Wang, Z. X., Li, S. M. \& Heide, L. Identification of the coumermycin $A_{1}$ biosynthetic gene cluster of Streptomyces rishiriensis DSM 40489. Antimicrob. Agents Chemother. 44, 3040-3048 (2000).

12 Zhang, X. \& Parry, R. J. Cloning and characterization of the pyrrolomycin biosynthesis gene clusters from Actinosporangium vitaminophilum ATCC 31673 and Streptomyces sp. strain UC 11065. Antimicrob. Agents Chemother. 51, 946-957 (2007).

$13 \mathrm{Mo}, \mathrm{S}$. et al. Elucidation of the Streptomyces coelicolor pathway to 2-undecylpyrrole, a key intermediate in undecylprodiginine and streptorubin B biosynthesis. Chem. Biol. 15, 137-148 (2008)

14 Williamson, N. R. et al. Biosynthesis of the red antibiotic, prodigiosin, in Serratia: identification of a novel 2-methyl-3-n-amyl-pyrrol (MAP) assembly pathway, definition of the terminal condensing enzyme, and implications for undecylprodiginine in biosynthesis in Streptomyces. Mol. Microbiol. 56, 971-989 (2005).

15 Kieser, T., Bibb, M. J., Buttner, M. J., Chater, K. F. \& Hopwood, D. A. Practical Streptomyces Genetics 405-420 (The John Innes Foundation, Norwich, 2000).

16 Sambrook, J. \& Russell, D. W. Molecular Cloning: A Laboratory Manual 1.1-1.162 (Cold Spring Harbor Laboratory, Cold Spring Harbor, New York, 2001).

17 Meurer, G. \& Hutchinson, R. Functional analysis of putative $\beta$-ketoacyl: acyl carrier protein synthase and acyltransferase active site motifs in a type II polyketide synthase of Streptomyces glaucescens. J. Bacteriol. 177, 477-481 (1995). 